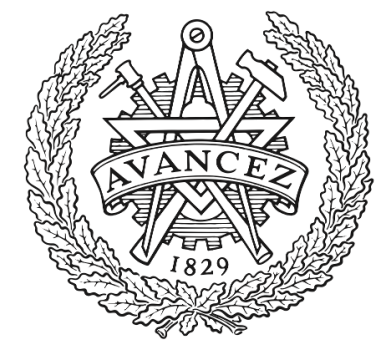

CHALMERS

UNIVERSITY OF TECHNOLOGY

\title{
Non-linear steering control for limit handling conditions using preview path curvature
}

Downloaded from: https://research.chalmers.se, 2023-04-26 09:43 UTC

Citation for the original published paper (version of record):

Klomp, M., Olsson, K., Sandberg, C. (2014). Non-linear steering control for limit handling conditions using preview path curvature. International Journal of Vehicle Autonomous Systems, 12(3): 266-283. http://dx.doi.org/10.1504/IJVAS.2014.063043

N.B. When citing this work, cite the original published paper. 


\title{
Non-linear steering control for limit handling conditions using preview path curvature
}

\author{
Matthijs Klomp* \\ Vehicle Dynamics CAE, \\ Volvo Car Group, \\ Gothenburg, Sweden \\ Email: matthijs.klomp@volvocars.com \\ *Corresponding author
}

\section{Klas Olsson}

Electrics/Electronics \& E-Propulsion (EEEP),

Volvo Car Group,

Gothenburg, Sweden

Email: klas.olsson@volvocars.com

\section{Carl Sandberg}

Vehicle Dynamics CAE, Volvo Car Group,

Gothenburg, Sweden

Email: carl.sandberg@volvocars.com

\begin{abstract}
Path-following steering control has many applications in autonomous driving, which may one day lead to cars running on autopilot. The developed controller addresses the path-following task by the required path curvature to reach a single preview point ahead of the vehicle as input to the controller. A pre-defined non-linear map of steering angle vs. curvature and vehicle speed is used to calculate the required steering angle. Calibration of the controller is done by performing a simple steady-state circle manoeuvre. The controller is evaluated using both simulations and experiments. Excellent path-following performance is achieved all the way up to the limit of adhesion. The actuation is smooth and the error rarely exceeds $1 \mathrm{~m}$ even at lateral acceleration levels of up to $10 \mathrm{~m} / \mathrm{s}^{2}$.
\end{abstract}

Keywords: path following; steering robot; steering control; vehicle dynamics; lateral control; limit handling.

Reference to this paper should be made as follows: Klomp, M., Olsson, K. and Sandberg, C. (2014) 'Non-linear steering control for limit handling conditions using preview path curvature', Int. J. Vehicle Autonomous Systems, Vol. 12, No. 3, pp.266-283.

Biographical notes: Matthijs Klomp holds a PhD degree in Vehicle Dynamics from Chalmers University of Technology and has over 15 years of experience within automotive engineering, particularly within chassis research and development, and vehicle dynamics and controls simulation. He currently works as a technical expert within vehicle dynamics simulation at Volvo Cars.

Klas Olsson received MSc in Systems, Controls and Mechatronics Engineering from Chalmers University of Technology. His thesis project, 'Preview Curvature 
Controller for a Passenger Car Steering Robot', was performed at Saab Automobile AB (2011). From 2011 to 2014 he worked at Volvo Cars within their Graduate Programme. During the program he worked with different part of automotive development; Complete Vehicle Concept Engineering, Electrical Energy Storage System development and Supplier Quality Management. He is now working as System Project Leader for High Voltage Batteries.

Carl Sandberg received MSc in Automotive Engineering, specialising in vehicle dynamics from Chalmers University of Technology, Sweden. His thesis project, 'Preview Curvature Controller for a Passenger Car Steering Robot', was performed at Saab Automobile. Has since graduation in 2011 has been working for Volvo Cars with vehicle dynamics attribute development through physical subjective and objective testing as well as CAE. He is responsible for coming vehicle dynamics simulator at Volvo Cars.

\section{Introduction}

Path-following steering control has many applications in autonomous driving (Hattori et al., 1992), which may one day lead to cars running on autopilot. Additionally, to complement human test drivers, using a robot to control steering during vehicle dynamics testing is useful (Tseng et al., 2005). Using a robot improves repeatability and the possibility to perform very precise manoeuvres, such as the sine-with-dwell manoeuvre (NHTSA, 2008). A robot is also capable of performing inputs faster than a human driver. This enables evaluation of not yet existing active safety systems.

Within this paper, the controller will be referred to as a closed-loop path controller. Longitudinal control of the vehicle, i.e. accelerating and braking, is not handled by the developed closed-loop controller. Similar controllers are often referred to as driver models when used in vehicle dynamic simulations (Plöchl and Edelmann, 2007). However, an important distinction from a classic driver model is that the closed-loop path controller aims at performing optimal vehicle control, with little consideration to human resemblance.

The controller shall follow a path at a wide range of speeds. This includes robust driving up to the limit of adhesion, and repeatability is more important than absolute path-following precision. The demands on the controller performance have been set to fulfil the needs of future users. In order to be integrated into the current user interface and combined with open-loop manoeuvres, certain demands are also set on the controller structure. These demands on path following are such that:

- A track shall be simple to define. The target track will be made up of several segments.

- All computations are to be performed in real time, i.e. closed-form expressions are preferred.

- The controller shall be able to cope with a wide range of operating conditions, such as speed, friction and levels of lateral acceleration.

- The controller shall be able to handle sudden disturbances, both to the input signals and to the vehicle itself. 
Many steering controllers are proposed in the literature. Examples include Edelmann et al. (2007) who use a layered approach with both feedback and compensatory feedforward based on the prediction from an advanced vehicle model. Other examples include Falcone et al. (2008) where model predictive control is proposed, using an advanced non-linear internal vehicle model to find the optimal future steering inputs. Most of these controllers rely on a preview error, heading error, distance error or multiple distance errors as in the case of Sharp et al. (2000). An issue with these feedback signals is, however, that there is no direct relationship between these factors and the steering angle. Similar to this paper, a kinematic relationship between the vehicle position and the target point is used by Kanayama et al. (1990). This paper, however, does not address the highly non-linear dynamics of the vehicle, required for robust tracking performance over the entire operating region of an automobile. Finally, how to parameterise the track and subsequently find which track segment must be tracked is not discussed in the abovementioned papers. In order to address the problems indicated above, a simple track segment identification scheme and the concept of preview curvature are devised in this paper. The preview curvature is here defined as the curvature of an arc that intersects the track at a pre-defined preview distance. The necessary steering angle to follow this preview curvature is subsequently obtained from a steady-state map created by a simple calibration manoeuvre. The novelty of this concept is thus the direct relationship between the required steering angle relative to the deviation from the target path and the lateral acceleration. A further contribution of this paper, unlike all of the above-mentioned papers, is that experiments in a real vehicle are performed to demonstrate the pathtracking capability of the proposed method.

The outline of the paper is such that first the necessary sensors and actuators (inputs/outputs) of the controller are described. Next, the structure of the controller is described in terms of how the track is defined, how the deviation from the track is determined and what the control strategy is to follow the track. Subsequently, path following and accuracy and stability and disturbance robustness are evaluated using software-in-the-loop simulation. Next, physical test results from path-tracking and lanechange tests are reported. Finally, conclusions are made and future work is identified.

\section{Sensors and actuators}

The steering controller in this paper uses feedback from the position, heading (yaw) angle, speed, yaw rate and lateral acceleration of the vehicle. For the sake of brevity, a planar surface is assumed in this work. For driving on banked curves and slopes, however, also this information must be incorporated.

As for the accuracy of the sensor information, it is obvious that the tracking accuracy of the controller is primarily dependent on the position accuracy of the positioning system. It is assumed that a $20-100 \mathrm{~Hz}$ Differential Global Positioning System (DGPS) is used for accurate speed and positioning. The yaw rate and lateral acceleration can readily be obtained using Inertial Measurement Unit (IMU). It is further recommended by the authors to use sensor fusion between the DGPS and IMU systems in order to improve the positioning accuracy, for instance, during poor satellite visibility, and to obtain an accurate heading angle of the vehicle. 
The control signal from the proposed controller is the steering wheel angle. In this paper, the required steering wheel angle is assumed to be achieved by a steering robot, but other actuators, such as the steering assist motor, are also possible.

\section{Track parameterisation}

Tracks are, in this paper, divided into lines and arcs as shown in Figure 1. Line segments are parameterised by the segment length, $L_{s}>0$. Arc segments are parameterised by the segment radius, $R_{s}>0$, and the angle $\theta$. The direction of the first segment is given by the angle $\gamma$. All subsequent track segments inherit the initial angle from end direction of the previous segment.

The starting point of the current segment $(k)$ is given by the coordinates $\left(x_{s}(k), y_{s}(k)\right)$. The current segment is defined by the segment within which the preview point lies.

Figure 1 Track parameterisation into line and arc segments (see online version for colours)

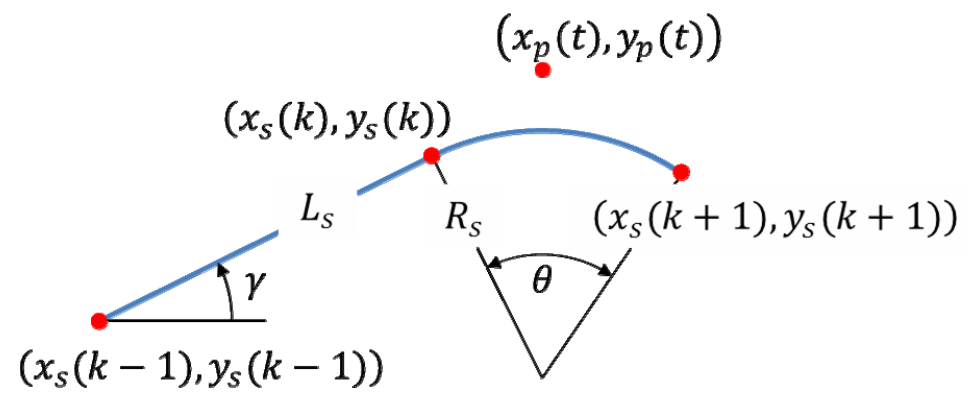

For line segments, the end point of the current segment (which also is the starting point of the next segment) is

$$
\left[\begin{array}{l}
x_{s}(k+1) \\
y_{s}(k+1)
\end{array}\right]=\left[\begin{array}{l}
x_{s}(k) \\
y_{s}(k)
\end{array}\right]+L_{s}\left[\begin{array}{c}
\cos \gamma \\
\sin \gamma
\end{array}\right]
$$

For arc segments, the end point of the arc is

$$
\left[\begin{array}{l}
x_{s}(k+1) \\
y_{s}(k+1)
\end{array}\right]=\left[\begin{array}{c}
x_{s}(k)-R_{s} \operatorname{sign} \gamma(\sin \gamma-\sin (\gamma+\theta)) \\
y_{s}(k)+R_{s} \operatorname{sign} \gamma(\cos \gamma-\cos (\gamma+\theta))
\end{array}\right]
$$

\section{Path control}

The basic structure of the controller is shown in Figure 2. Each part of the controller will be described in detail in the following subsections. 
Figure 2 Basic structure of the controller (see online version for colours)

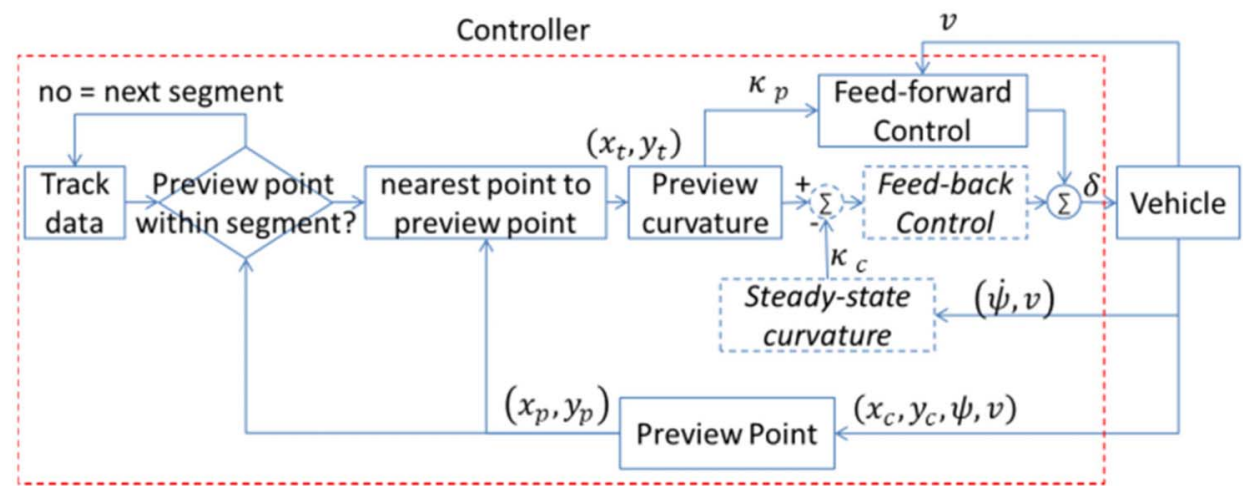

The controller consists of an outer loop and an optional inner loop. The outer loop computes the preview curvature shown in Figure 3, which is the reciprocal of the radius required to reach the preview point. Before the computation of the preview curvature, a non-trivial check to ascertain if the preview point is within the current segment is performed.

Figure 3 Concept of preview curvature (see online version for colours)

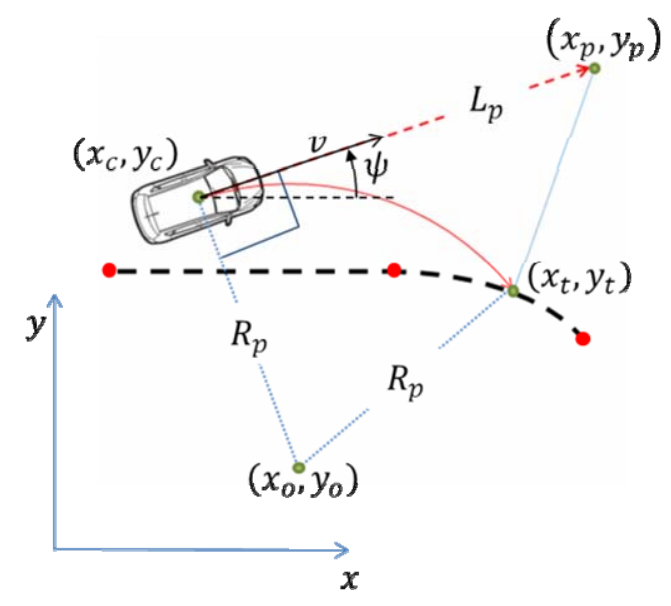

Apart from the steering angle, computed by the feed-forward control, an optional innerloop feedback control is added. This inner-loop feedback control is only needed to eliminate a steady-state error in the control when the track curvature does not change for a long time. One example of such a situation is steady-state circular driving.

\subsection{Preview point}

Since the vehicle needs time to react to changes in the track and/or deviations from the track, the distance to the track at a preview distance from the vehicle is used as a control reference. 
This preview point, shown in Figure 3, is a point along the heading direction at a speed-dependent look-ahead distance from the vehicle:

$$
\left[\begin{array}{l}
x_{p} \\
y_{p}
\end{array}\right]=\left[\begin{array}{l}
x_{c} \\
y_{c}
\end{array}\right]+L_{p}\left[\begin{array}{l}
\cos \psi \\
\sin \psi
\end{array}\right]
$$

where

$$
L_{p}=L_{p 0}+\tau_{p} v
$$

where $L_{p 0}$ is the minimum preview distance, $\tau_{p}$ is the preview time and $v$ is the vehicle speed.

\subsection{Is the preview point within segment?}

Prior to determining the distance of the preview point to the track, the controller checks if the preview point is within the current segment. This procedure is different depending on whether the segment is a line or an arc segment. If the preview point is not within the current segment, the next subsequent segment is checked. The computation of deviation from the preview point to the track can then be performed on the correct segment.

\subsubsection{Line segments}

For line segments, the preview point is within the current line if

$$
0 \leq L_{t} \leq L_{s}
$$

where

$$
L_{t}=\left(x_{p}-x_{s}\right) \cos \gamma+\left(y_{p}-y_{s}\right) \sin \gamma
$$

i.e. the projected length of the preview point relative to the starting point of the segment.

\subsubsection{Arc segments}

Knowing if the preview point lies within an arc segment is less straightforward than for line segments, and two projections instead of one is required. For this purpose, we first create the unit vectors $\boldsymbol{u}$ and $\boldsymbol{v}$ shown in Figure 4. With these unit vectors, we can locate the position of the preview point $\left(x_{p}, y_{p}\right)$ from equation (3) relative to the arc endpoint $\left(x_{s}, y_{s}\right)$ from equation (2) and the arc radius $R_{s}$ as the vector $\boldsymbol{w}$, which is

$$
\boldsymbol{w}=\left[\begin{array}{c}
x_{p}-x_{s} \\
y_{p}-y_{s}
\end{array}\right]-R_{s} \boldsymbol{u}
$$

where

$$
\boldsymbol{u}=\operatorname{sign} \theta\left[\begin{array}{c}
\sin \gamma \\
-\cos \gamma
\end{array}\right]
$$


Figure 4 Preview point segment check for arc segments (see online version for colours)

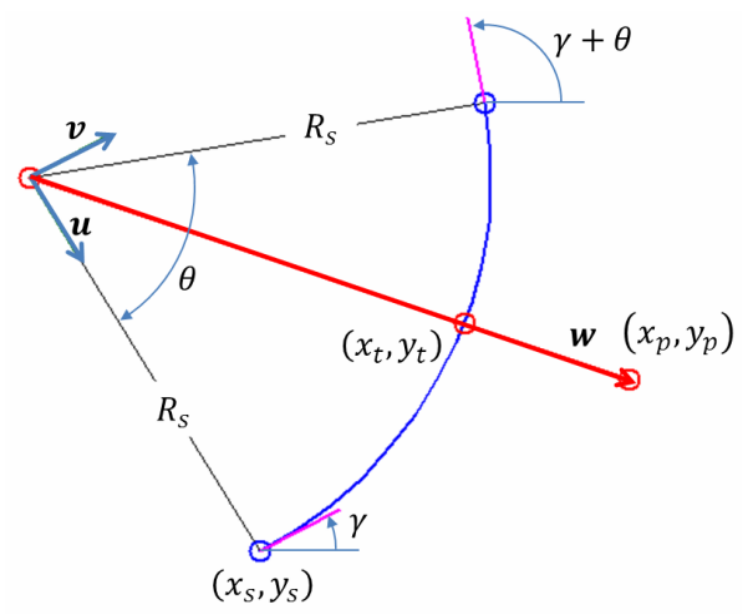

Projections of the vector $\boldsymbol{w}$ onto the unit vectors $\boldsymbol{u}$ and $\boldsymbol{v}$ are used to check if the preview point lies within the arc segment. The first projection is compared to the length of the vector $\boldsymbol{w}$ relative to the angle $\theta$ of the arc. An additional check is whether the vector $\boldsymbol{w}$ lies in the direction of vector $v$ or opposite to it. Different conditions apply for segments which are smaller than a half circle and those that are larger. Now, the preview point is within an arc segment with $|\theta|<\pi$ if

$$
\boldsymbol{w} \cdot \boldsymbol{u}<|\boldsymbol{w}| \cos \theta \wedge \boldsymbol{w} \cdot \boldsymbol{v}>0
$$

where

$$
\boldsymbol{v}=\left[\begin{array}{l}
\cos \gamma \\
\sin \gamma
\end{array}\right]
$$

For segments where $\pi \leq|\theta|<2 \pi$, the preview point is within the arc segment if

$$
\boldsymbol{w} \cdot \boldsymbol{u}>|\boldsymbol{w}| \cos \theta \vee \boldsymbol{w} \cdot \boldsymbol{v}>0
$$

In equations (9) and (11), $\wedge$ denotes a conjunction (AND) and $\vee$ a disjunction (OR).

\subsection{Nearest point on the track from the preview point}

Next, the nearest point from the preview point to the track is found by building on the previous calculations. The preview curvature, used for the steering control, is the curvature necessary to reach this point.

The nearest point from the preview point to a line segment is

$$
\left[\begin{array}{l}
x_{t} \\
y_{t}
\end{array}\right]=L_{t}\left[\begin{array}{c}
\cos \gamma \\
\sin \gamma
\end{array}\right]
$$


where $L_{t}$ is computed in Section 4.2.1.

The nearest point from the preview point to an arc segment is

$$
\left[\begin{array}{l}
x_{t} \\
y_{t}
\end{array}\right]=R_{s} \frac{\boldsymbol{w}}{|\boldsymbol{w}|}
$$

where $R_{s}$ and $\boldsymbol{w}$ are shown in Figure 4.

\subsection{Preview curvature}

The preview curvature (see Figure 3) is obtained by knowing that

$$
\left[\begin{array}{l}
x_{0} \\
y_{0}
\end{array}\right]=\left[\begin{array}{l}
x_{c} \\
y_{c}
\end{array}\right]+R_{p}\left[\begin{array}{c}
\sin \psi \\
-\cos \psi
\end{array}\right]
$$

and that

$$
\left(x_{c}-x_{0}\right)^{2}+\left(y_{c}-y_{0}\right)^{2}=\left(x_{t}-x_{0}\right)^{2}+\left(y_{c}-y_{0}\right)^{2}
$$

Combining (14) and (15) gives

$$
\kappa_{p}=\frac{1}{R_{p}}=2 \frac{\left(x_{c}-x_{t}\right) \sin \psi-\left(y_{c}-y_{t}\right) \cos \psi}{\left(x_{c}-x_{t}\right)^{2}+\left(y_{c}-y_{t}\right)^{2}}
$$

which contains only known parameters obtained from our previous calculations. Next is applying the right steering input to follow the preview curvature. As before, this curvature can be interpreted as the curvature necessary to reach the track within the given preview distance.

\subsection{Feed-forward control}

The steering wheel angle which is necessary to reach the preview curvature is

$$
\delta_{\mathrm{ff}}=i_{S} l \kappa_{p}+i_{S} \mu g K_{U} \operatorname{atanh} \frac{\kappa_{p} v^{2}}{\mu g}
$$

for

$$
\kappa_{p} v^{2}<\mu g
$$

where $i_{S}$ is the steering ratio, $l$ the wheel base, $K_{U}$ the understeer gradient, $\mu$ the surface friction and $g$ the gravitational acceleration. These parameters can, for instance, be found by a parameter identification from a steady-state circular driving manoeuvre (ISO 4138, 2004).

Linearising the above expression, we arrive at the familiar steady-state steering expression for a linear bicycle model:

$$
\delta_{\mathrm{ff}}=i_{S}\left(l+K_{U} v^{2}\right) \kappa_{p}
$$


Both expressions are drawn in Figure 5 where the solid line corresponds to equation (17) and the dashed line to equation (19).

Figure 5 Steering as function of lateral acceleration (see online version for colours)

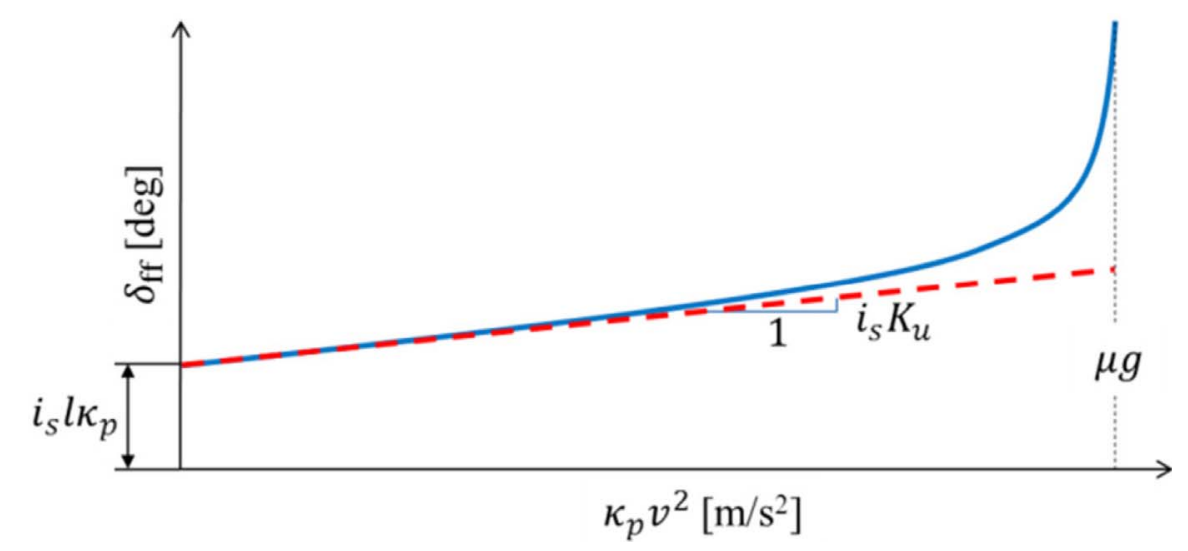

\subsection{Feedback control}

For the optional inner-loop feedback control, the current steady-state yaw rate is used:

$$
\kappa_{c}=\frac{\dot{\psi}}{v}
$$

The control error then becomes

$$
e=\kappa_{p}-\kappa_{c}
$$

The controller is designed as a PI controller with anti-windup ( $\AA$ ström, 2002).

\subsection{Preview distance and corner cutting}

For the Preview Curvature Controller, the only tuning parameter is the preview time. The resulting reference curvature calculated, as described in the previous section, becomes larger as preview time is decreased. The track curvature changes in steps, because lines and arcs are used to define the track, meaning that a short preview time will lead to a rapid change in the desired curvature. Since the preview point enters the curve before the vehicle does, the preview curvature will cut the corner with a large radius starting before the track bend begins. A long preview will hence lead to a smoother controller, but also to a larger corner cutting and larger deviations from the reference track (see Figure 6). The user has to make the trade-off between precision and smoothness by tuning the preview time.

Again, the benefit of this corner cutting is that the preview curvature increases smoothly up to the curvature of the curve when approaching a curve. That is, the preview curvature (and the corresponding steering angle) changes smoothly even though the curvature of the track changes step-wise. 
Figure 6 Corner cutting because of preview (see online version for colours)

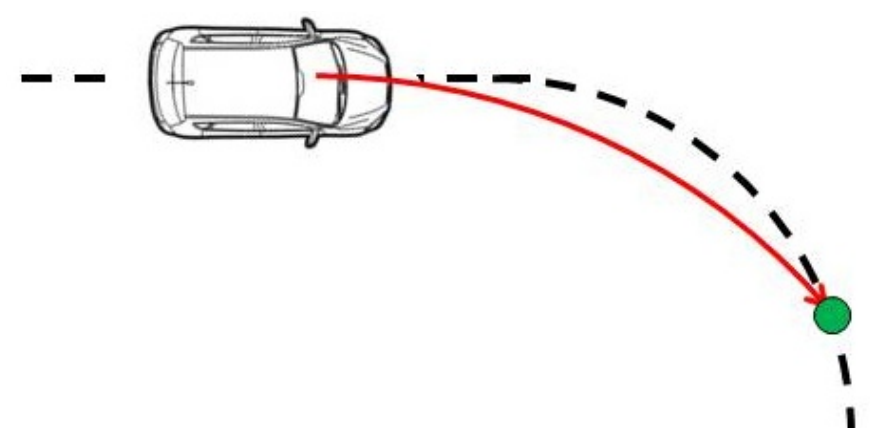

\section{Simulation results}

This section presents the results from simulations of track driving and the control of specific manoeuvres. The purpose of these simulations was to determine the influence of the preview time on the tracking accuracy vs. stability of the control and to understand the robustness of the controller to changes in the track, road conditions and external disturbances.

\subsection{Effect of preview time on precision vs. stability}

The track driving simulations in IPG CarMaker (Wittenburg, 2008) have been performed on the Hockenheim track using IPG Driver for longitudinal control.

As the velocity of the car will be controlled by a human driver when testing the controller in a vehicle, IPG Driver was used for longitudinal control in simulations. As IPG Driver is completely decoupled from the steering controller, it will not perform very well, meaning that the car might be braking or accelerating while turning and thereby at times destabilising the vehicle. This is a weakness in the simulations although the disturbances caused by the longitudinal controller do test the ability of the steering controller to stabilise the vehicle with such disturbances. The destabilising behaviour of the longitudinal controller can also be mitigated by lowering the acceleration limits in IPG Driver resulting in more careful driving in curves.

As mentioned above, there is a trade-off between the tracking error and control signal smoothness as function of the preview time. The simulations shown in this section aim at determining the effects of the preview time on these two parameters. Subsequently, a subjective evaluation of the control signal activity is made to determine the desired preview time.

Figure 7 shows the effect of changing the preview time on deviation from the centre line of the track. With 0.6 seconds' preview time, the controller is oscillating around the reference path, as can be seen in Figures 7 and 8. Figure 9 shows how the mean deviation from the centre line increases with preview time. 
Figure 7 Deviation from centre of track vs. time. Notice the difference in the scale of the $y$ axis; the deviation from the centre line is approximately four times larger with the longest preview time compared to the shortest. IPG driver is set to drive at a constant speed, $50 \mathrm{~km} / \mathrm{h}$ (see online version for colours)
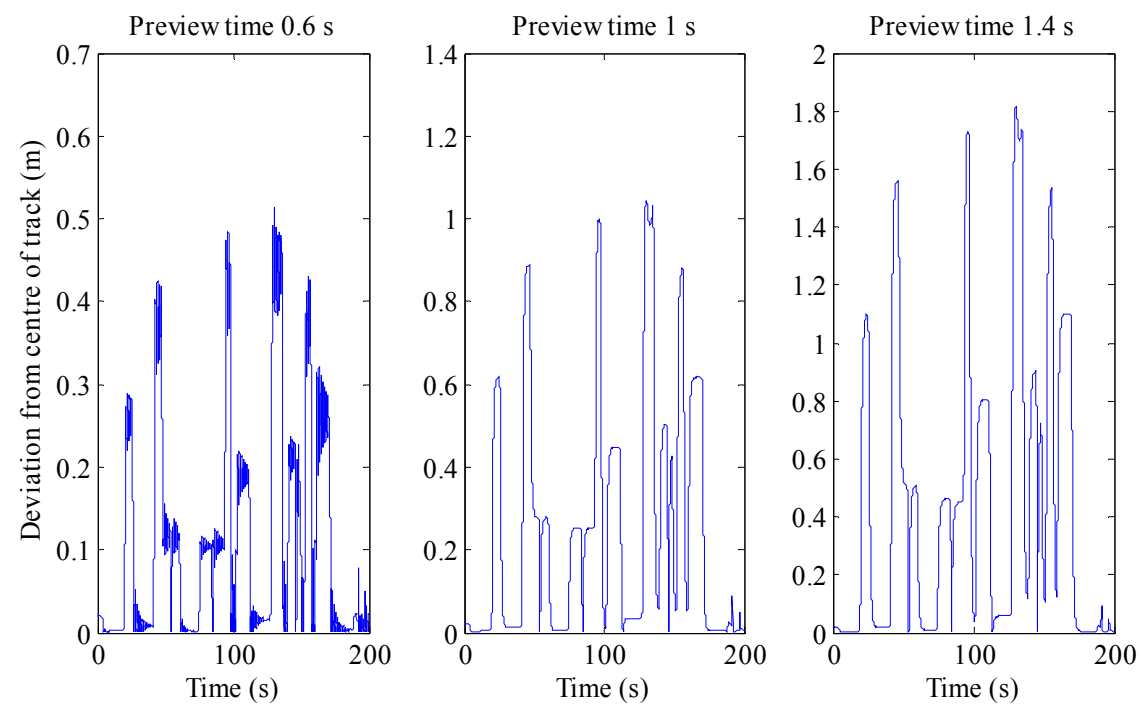

Figure 8 Steering angle over time for different preview times. At 0.6 seconds' preview time, the steering wheel oscillates considerably (see online version for colours)
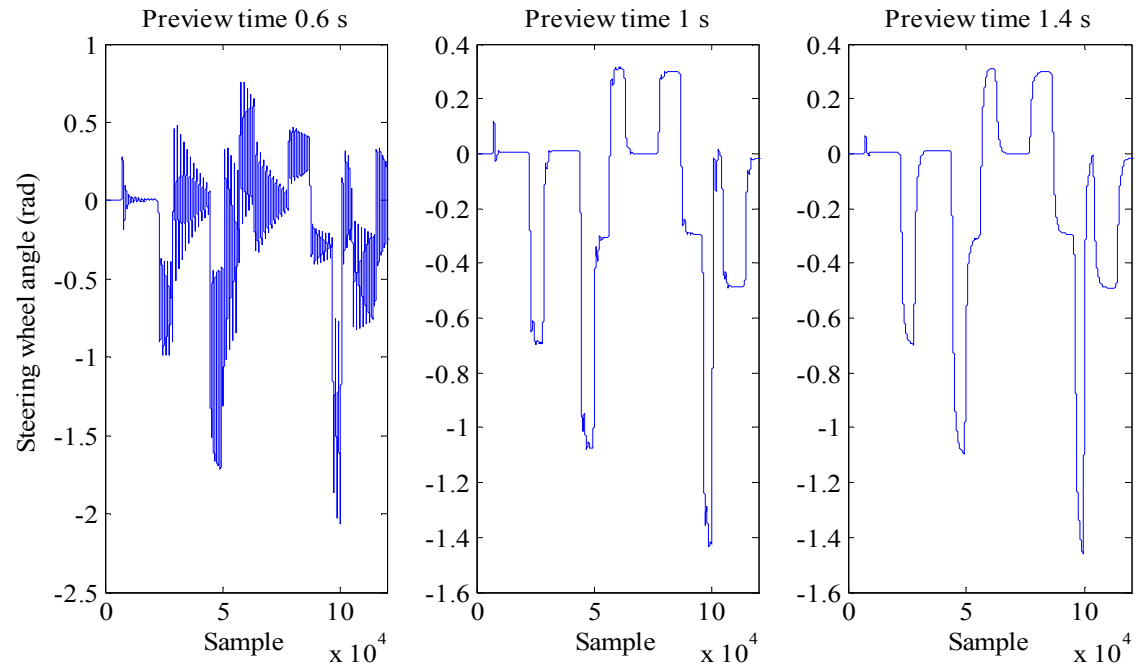

From the simulation results shown in Figures 7-9, it can be seen that there is a trade-off between steering oscillation and corner cutting, resulting in track deviation. For this work, it was decided that a preview time of 0.8 seconds gave a good path-following performance without excessive control signal activity. 
Figure 9 Mean deviation from track centre line at different preview times. The mean deviation increases almost linearly with preview time (see online version for colours)

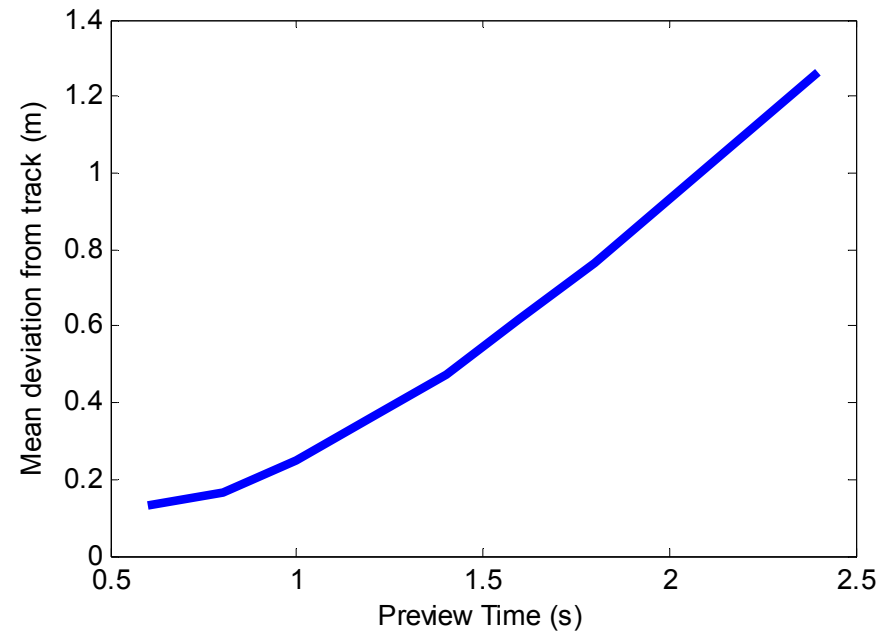

\subsection{Steady-state circle results}

The cascaded steady-state cornering controller introduced above has been tuned and evaluated only in simulations. The reason for evaluating only the controller in simulations was the lack of access to a large enough vehicle handling area to perform the steady-state cornering manoeuvre. However, the resulting gain schedule for required steering gain generated from the steady-state cornering simulations have been used with good result when evaluating the track driving controller. The performance (the radius error) of the steady-state circle controller is shown in Figure 10. The highest integrator gain $\left(K_{i}=0.01\right)$ in the figure is the highest gain that does not make the vehicle unstable.

Figure 10 Radius error over time for different integrator gains $\left(K_{i}\right)$. Further increasing the gain causes the steering to oscillate. Only an approximated value of the steering gain and wheel base is used for the preview gain (see online version for colours)

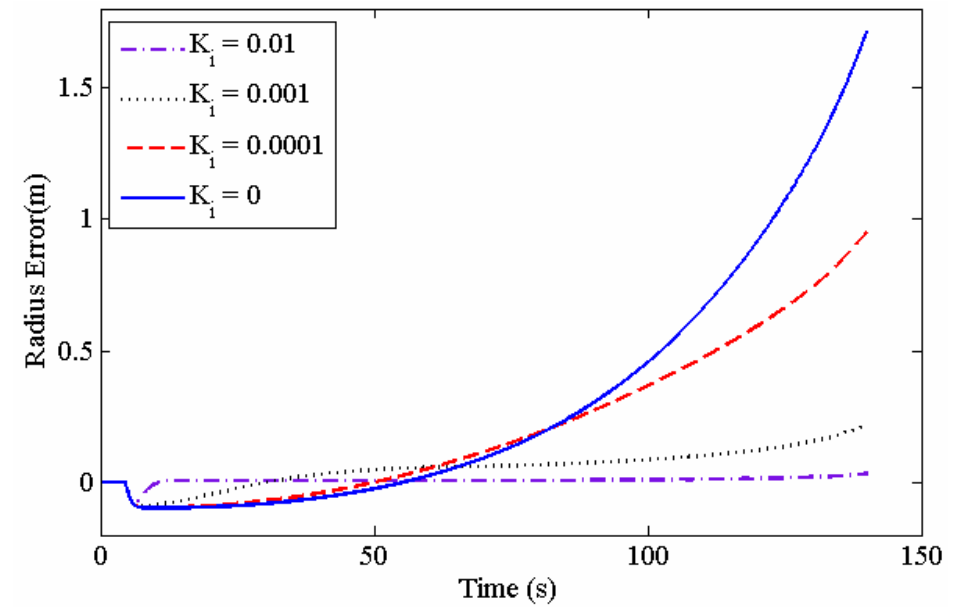




\section{Experimental results}

The experimental testing of the controller has been made at the Råda Air Base (shown in Figure 11), which is a decommissioned air field. The Råda Air Base suits the needs for the evaluation of the controller since it is off-limits for general traffic and is also an open field with minimum disturbances to the GPS signal. The track shown in Figure 11 has a long straight, a couple of narrow turns and a large radius curve to enable evaluation of the controller performance in all these situations.

The vehicle used in the validation is a four-wheel-driven MY'09 Saab 9-5 sedan with a 190 HP diesel engine.

The preview time used in all physical tests is 0.8 seconds, as in the simulations. Although it is not given that the preview distance found in simulations also gives the desired performance in vehicle tests, simulations can be used to find a good starting value for the preview time. Additionally, the minimum preview distance was limited to 10 metres in order to limit the hand wheel angles at low speed.

Figure 11 The track driven at Råda Air Base (see online version for colours)

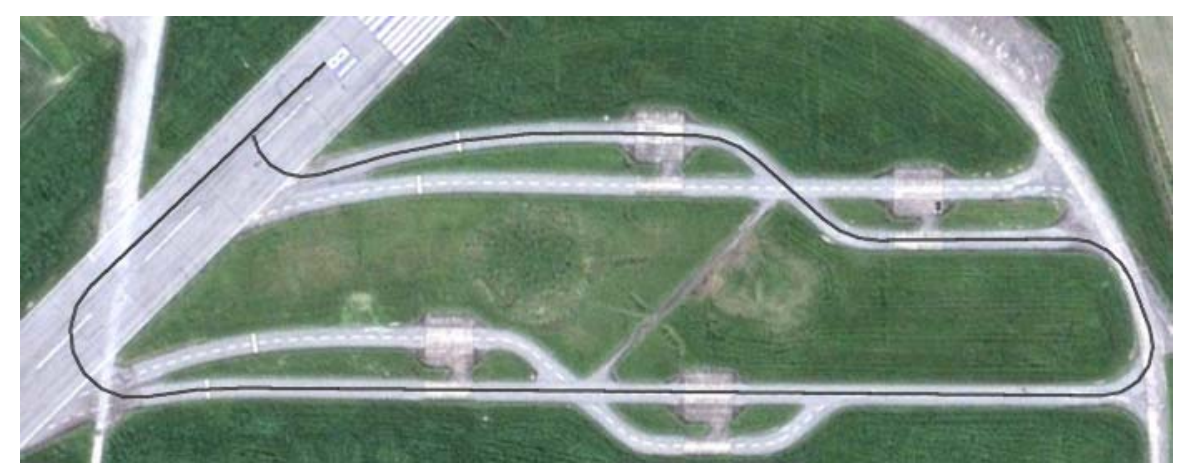

\subsection{The steering robot}

The steering control is performed by a steering robot which consists of an electric drive unit that is mounted at the windscreen of the vehicle using vacuum cups. The drive is connected to the original steering wheel via a geared ring mounted behind the original steering wheel. The steering wheel can be operated as usual when the robot is not active and therefore the vehicle can be controlled by a driver until the start of a robot-controlled manoeuvre. The robot is controlled by a real-time computer running a real-time operating system. The real-time computer generates a control signal which is transferred into a pulse-width-modulated signal by power electronics fed with power from the 12-volt system of the vehicle.

The robot is programmed by the test engineer using a windows-based graphical user interface running on an external computer. The real-time computer also has a CAN interface which can be used for connection of external sensors. The two main inputs to the controller, the position and heading angle of the vehicle, are measured by a GPS system and an IMU, respectively. The speed of the vehicle in its course direction is also measured by the GPS. The GPS system used for the experiments is a VBOX III system 
from RaceLogic. It has an update frequency of $100 \mathrm{~Hz}$ and provides the location of the receiver as well as an exact velocity measurement. The IMU which was used is an iDis-FMS IMU with optical gyros from iMAR GmbH.

To improve the position measurement from the GPS, measurements from the IMU are taken into account using sensor fusion (see Olsson and Sandberg, 2011, for more details).

\subsection{Precision}

The precision of the controller is very high when driving on straight segments. In Figure 12, for instance, it can be seen that the deviation on the long straight segment (from 15 to 25 seconds) is below 0.1 metres. When cornering, the preview causes the car to cut the corners (Figure 13). However, the vehicle still stays within 1.2 metres from the centre line of the track at all times. In longer bends, as the one opposite to the long straight in Figure 11, the deviation from the centre line decreases over time, as seen in Figure 12 (between approximately 53 and 57 seconds).

Figure 2 The deviation from the track during one lap of the Råda track. The deviation is shown relative to the lateral acceleration of the vehicle. The speed was high, exceeding $100 \mathrm{~km} / \mathrm{h}$ on the straight. The speed was kept high enough to reach lateral accelerations of $8-10 \mathrm{~m} / \mathrm{s}^{2}$ in all bends (see online version for colours)

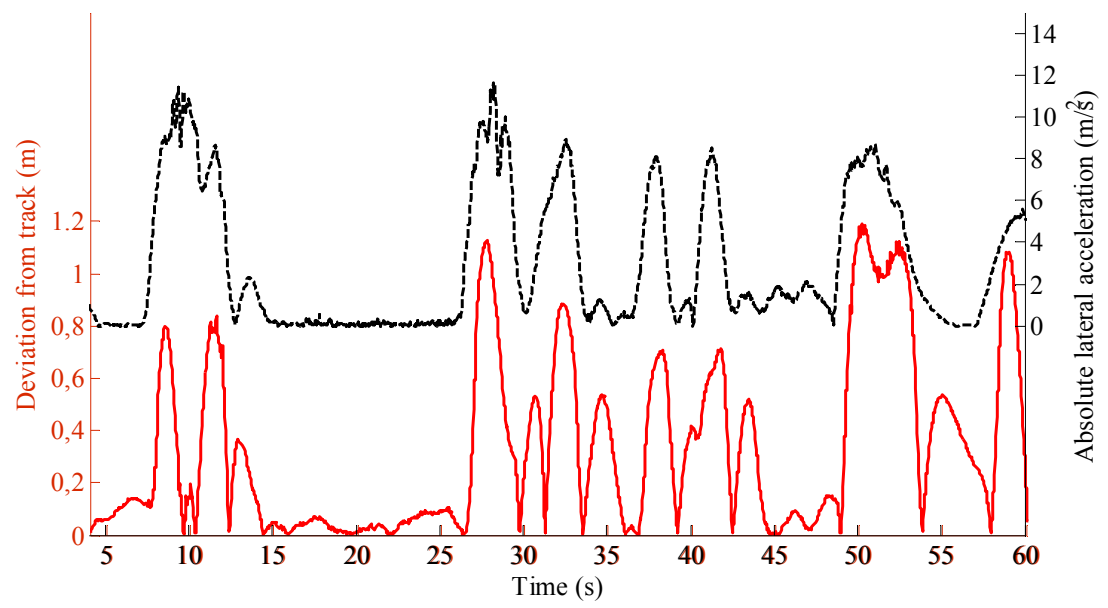

\subsection{Control signal activity}

The control signal has proven to be very smooth during the physical testing. In Figure 14, the control signal from the same lap around the Råda track from which the data presented in Figure 12 were recorded is shown. As seen in the left plot of Figure 14, the control signal is smooth and the mechanics of the actuator can in the right close-up be seen to filter out the noise in the control signal. 
Figure 13 Vehicle position relative to the track when cornering at high speed. The lateral acceleration of the vehicle is between 0.8 and $1 \mathrm{~g}$. Preview time is 0.8 seconds, with a minimum preview distance of 15 metres. The difference in path is due to different speeds; the path closest to the track is driven at the highest speed with the front tyres entering saturation

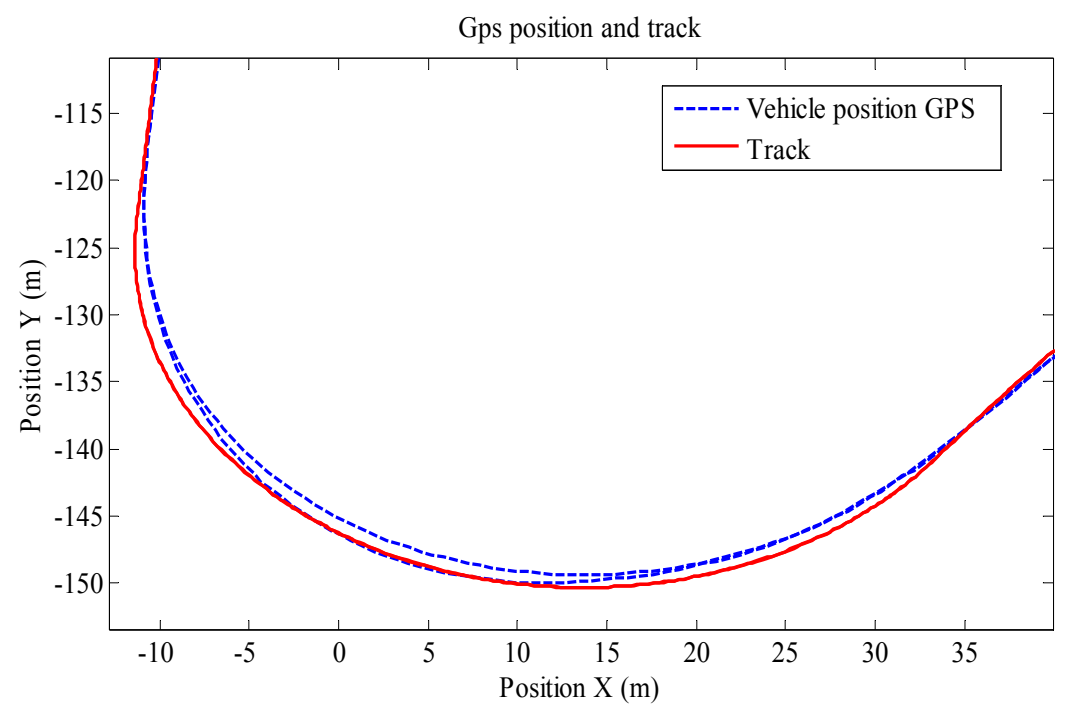

Figure 14 Control signal during one lap on the Råda track. The peaks in steering wheel angle are a result of saturated front wheels giving a rapid increase in requested angle (see online version for colours)

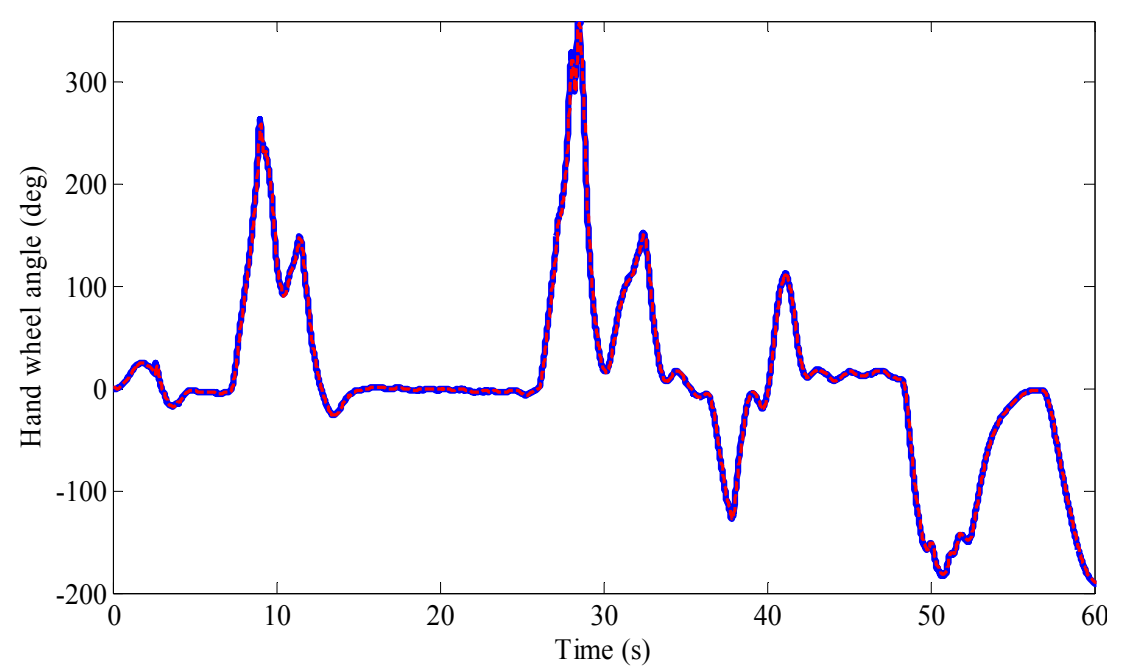




\subsection{Double lane-change test}

Although the double lane-change manoeuvre has not been considered specifically during the development, a brief test was made using the standard Preview Curvature Controller. During the test, the car was equipped with all-season tyres. The cones were positioned roughly as the ISO-17387 lane-change standard.

The most important result from the lane-change test is that the controller proved to execute very repetitive and precise control. The maximum possible entry speed was $65 \mathrm{~km} / \mathrm{h}$ limited by the chosen all-season tyres. Figure 15 shows 13 runs of the lane change with speeds from 45 to $65 \mathrm{~km} / \mathrm{h}$. Although the front tyres partly saturated in the first turn at $65 \mathrm{~km} / \mathrm{h}$, it can be seen from Figure 15 that the repeatability is still very good. Once the reference track had been adjusted to achieve a good path through the cones, the controller drove nearly the same path at all tested speeds, up to the lateral grip limit.

Figure 15 Lane-change test. The figure shows 13 runs with speeds from 45 to $65 \mathrm{~km} / \mathrm{h}$ (see online version for colours)

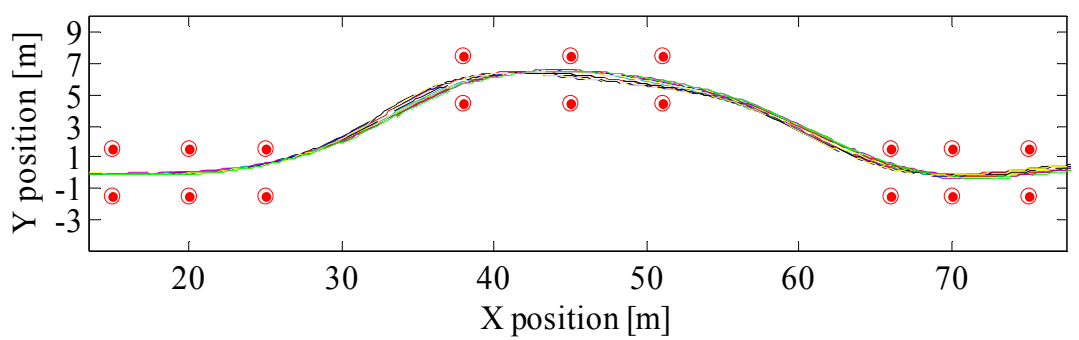

\section{Discussion}

The developed Preview Curvature Controller shows good performance and definitely fulfils the aims and demands of the paper. As discussed briefly above, the controller will not give absolute precision, due to corner cutting. However, since it is developed for driving at a track with margins, the advantage of a more smooth control, due to corner cutting, is deemed more important than the track deviation of up to 1.5 metres. As long as the tyres are not saturated, 1.5 metres has been the largest error observed during testing.

Also, instead of absolute precision, repeatability is a more important performance criterion of the controller. The repeatability of the presented controller was demonstrated in the previous section as it was possible to perform a double lane change at almost as high speed as an experienced test driver could perform it $(65 \mathrm{~km} / \mathrm{h})$ after only 30 minutes of adjusting the reference track. The speed itself is not what was found most impressive, but rather that the same path was followed in all test runs, which is an important difference to human drivers.

The developed controller does have very good performance and would be ready for use as designed. However, before commercialisation, it would be necessary to add more safety functions for system failures, etc. Furthermore, the coupling with a longitudinal vehicle controller and use of other measurement systems are necessary to study in order to commercialise the controller. 
An obvious extension of the lateral vehicle controller presented in this paper is to couple it with longitudinal control. Such a longitudinal controller would obviously require additional actuators, such as pedal actuators.

Creating a reference track relative to a physical track to be followed was shown to be a rather time-consuming task in the paper. The task was performed by using an aerial photo of the track and then measuring the radius and degrees of the turns and the length of the straights. To make this task more time-efficient, either an automated way of creating a track from a picture or a function to rerun a manually driven track should be implemented.

\section{Conclusions}

The controller developed during this paper uses a new method of feedback path control which continuously changes the reference signal based upon vehicle location. The controller calculates the curvature required to arrive at a preview point on a pre-defined track ahead of the vehicle. Compared to the related steering controllers, found in previous research, the newly developed controller uses a more straightforward way of calculating controller inputs from track deviation and vehicle states. The controller also uses a simple yet effective way of, given a reference input, calculating the required steering angle using an exponential function. Additionally, a method to parameterise the track and find out on which segment of the track the preview point is located was developed.

The absence of multiple controller inputs gives a great advantage since it is easy to analyse and understand why the controller behaves as it does, and how to customise it for specific demands that may differ from the current ones. For example, the fact that the parameterisation of the controller consists of physical units such as steering ratio, wheel base, understeer gradient and maximum lateral acceleration and preview time also makes the controller intuitive and easy to calibrate to different cars and tracks.

Despite its relative simplicity and low computational demand, the Preview Curvature Controller shows very good performance both in simulations and in physical testing. Driving on a narrow track at high speed, the deviation from the defined track has been kept below 1.5 meters, but more importantly, the repeatability is close to absolute. It has also been proven to be stable for disturbances to input signals and to the vehicle itself. The actuation of the steering has during the testing been perceived to be smooth by the operators.

\section{Acknowledgements}

The main part of this work was performed at the Chassis Department at Saab Automobile in Trollhättan, Sweden, in cooperation with Chalmers University of Technology in Gotenburg. The authors would like to thank Mathias Lidberg from Chalmers for academic support, and Modelon AB and IPG Automotive $\mathrm{GmbH}$ for providing CarMaker licences. 


\section{References}

Åström, K.J. (2002) Control System Design, Lecture Notes for ME 155A, University of California, Santa Barbara, CA

Edelmann, J., Plöchl, M., Reinalter, W. and Tieber, W. (2007) 'A passenger car model for higher lateral accelerations', Vehicle System Dynamics, Vol. 45, No. 12, pp.1117-1129.

Falcone, P., Eric Tseng, H., Borrelli, F., Asgari, J. and Hrovat, D. (2008) 'MPC-based yaw and lateral stabilisation via active front steering and braking', Vehicle System Dynamics, Vol. 46, Supplement 1, pp.611-628.

Hattori, A., Hosaka, A., Taniguchi, M. and Nakano, E. (1992) 'Driving control system for an autonomous vehicle using multiple observed point information', Proceedings of the Intelligent Vehicles '92 Symposium, 29 June-1 July, Detroit, MI, pp.207-212.

ISO 4138 (2004) Passenger Cars: Steady-State Circular Driving Behaviour - Open-Loop Test Methods, International Organization for Standardization, Geneva, Switzerland.

Kanayama, Y., Kimura, Y., Miyazaki, F. and Noguchi, T. (1990) 'A stable tracking control method for an autonomous mobile robot', Proceedings of the IEEE International Conference on Robotics and Automation, 13-18 May, Cincinnati, OH, pp.384-389.

NHTSA (2008) TP-126-02: Laboratory Test Procedure for FMVSS 126, Electronic Stability Control Systems, NHSTA, Washington, DC.

Olsson, K. and Sandberg, C. (2011) The Preview Curvature Controller for a Passenger Car Steering Robot, Chalmers University of Technology, Gothenburg.

Plöchl, M. and Edelmann, J. (2007) 'Driver models in automobile dynamics application', Vehicle System Dynamics: International Journal of Vehicle Mechanics and Mobility, Vol. 45, Nos. 7-8, pp.699-741.

Sharp, R.S., Casanova, D. and Symonds, P. (2000) 'A mathematical model for driver steering control, with design, tuning and performance results', Vehicle System Dynamics, Vol. 33, No. 5, pp.289-326.

Tseng, H., Asgari, J., Hrovat, D., van der Jagt, P., Cherry, A. and Neads, S. (2005) 'Evasive manoeuvres with a steering robot', Vehicle System Dynamics, Vol. 43, No. 3, pp.199-216.

Wittenburg, J. (2008) Dynamics of Multibody Systems, 2nd ed., Springer, Berlin. 